\title{
Comparação de métodos para avaliar a adaptabilidade e estabilidade produtiva em algodoeiro
}

\author{
João Luís da Silva Filho(1), Camilo de Lelis Morello(2), Francisco José Correia Farias ${ }^{(3)}$, Fernando Mendes Lamas ${ }^{(4)}$, \\ Murilo Barros Pedrosa ${ }^{(5)}$ e José Lopes Ribeiro(6)
}

\begin{abstract}
(1)Embrapa Algodão, Caixa Postal 174, CEP 58107-720 Campina Grande, PB. E-mail: joaoluis@cnpa.embrapa.br (2)Embrapa Algodão - Núcleo Goiás, BR 153, Km 4, CEP 74001-970 Goiânia, GO. E-mail: morello@cnpa.embrapa.br (3)Embrapa Algodão - Núcleo do Mato Grosso, Rua Poxoréu, no 612, CEP 78750-000 Primavera do Leste, MT. E-mail: farias@cnpa.embrapa.br (4)Embrapa Agropecuária Oeste, Caixa Postal 661, CEP 79804-970 Dourados, MS. E-mail: lamas@cnpao.embrapa.br (5)Fundação Bahia, Avenida Ahylon Macedo, no 11, Morada Nobre, CEP 47806-180 Barreiras, BA. E-mail: fundacaoba.algodao@aiba.org.br ${ }^{(6)}$ Embrapa Meio-Norte, Caixa Postal 01, CEP 64006-220 Teresina, PI. E-mail: jlopes@cpamn.embrapa.br
\end{abstract}

\begin{abstract}
Resumo - O objetivo deste trabalho foi comparar diferentes métodos de estimação da adaptabilidade e estabilidade produtiva em 17 genótipos de algodoeiro avaliados em 23 ambientes do Cerrado brasileiro. Os efeitos de genótipos e ambientes e a interação genótipos $\mathrm{x}$ ambientes foram significativos. Os modelos da ecovalência e AMMI indicaram a cultivar BRS Cedro como a mais estável, enquanto as cultivares Delta Penta e BRS Ipê foram identificadas como as mais instáveis; nenhuma delas esteve entre as mais produtivas. De acordo com o método de Eberhart \& Russell, Lin \& Binns e Annicchiarico, as cultivares BRS 269 - Buriti e FMT 701 e o genótipo CNPA GO 2001-999 foram os mais indicados para plantio no Cerrado, e se destacaram entre as cinco mais produtivas na média dos ambientes. A identificação de adaptabilidades específicas, proporcionada pela análise AMMI, é de grande relevância no estudo do comportamento dos genótipos. Pelo conjunto de informações obtidas e pela facilidade de uso e interpretação, recomenda-se o emprego do método de Lin \& Binns, que pode ser complementado pela análise AMMI.
\end{abstract}

Termos para indexação: Gossypium hirsutum, análise AMMI, avaliação de cultivares, ecovalência, interação genótipos $\mathrm{x}$ ambientes.

\section{Comparison of methods for the evaluation of adaptability and stability for yield in cotton genotypes}

\begin{abstract}
The objective of this work was to compare different methods used to estimate adaptability and stability of 17 cotton genotypes evaluated in 23 locations of the Brazilian savannah. Genotype and environment effects and genotype $\mathrm{x}$ environment interaction were significant. According to ecovalence and AMMI models, cultivar BRS Cedro showed the best stability. Varieties Delta Penta and BRS Ipê were among the most unstable genotypes, but not among the most productive. Using the methods of Eberhart \& Russel, Lin \& Binns and Annicchiarico, genotypes BRS 269 - Buriti, FMT 701 and CNPA GO 2001-999 were the most stable and among the five most productive on average. The evaluation of the specific adaptabilities provided by the AMMI analysis is of great importance in the study of the behavior of genotypes. The amount of information generated and the facilities of interpretation favors Lin \& Binns method, which can be complemented by an AMMI analysis.

Index terms: Gossypium hirsutum, AMMI analysis, assesment of cultivars, ecovalence, genotype x environment interaction.
\end{abstract}

\section{Introdução}

No Brasil, o algodoeiro (Gossypium hirsuntum L.) é cultivado em toda a faixa tropical, e a região do Cerrado se destaca tanto em área cultivada quanto em produtividade. Contudo, dada a extensão dessa região, condições diferentes de clima e solo são encontradas e, portanto, é esperada ausência de comportamento consistente de genótipos nos diversos ambientes.
A identificação de cultivares com maior estabilidade fenotípica é uma das estratégias para amenizar o efeito da interação genótipos x ambientes (GxA).

A metodologia denominada de ecovalência, proposta por Wricke, citado por Cruz \& Regazzi (1997), decompõe a soma de quadrado da interação em partes atribuídas a cada genótipo, e considera como mais estável o genótipo que apresente menor estimativa para a ecovalência. Ramalho et al.(1993) comentam que a ecovalência é 
uma das formas de se medir a estabilidade no sentido agronômico, em que são considerados como genótipos mais estáveis os que acompanham o desempenho médio dos ambientes. Estudos de estabilidade do algodoeiro em condições de Cerrado com uso da ecovalência foram realizados por Farias (2005) e Souza et al. (2006).

Entre as metodologias que fazem uso da análise de regressão, a proposta por Eberhart \& Russell (1966) é uma das mais difundidas. Baseia-se em uma regressão linear simples dos genótipos em função de índices ambientais. Os autores sugerem o coeficiente de inclinação da reta como medida de adaptabilidade e os desvios da regressão como medida de estabilidade, e consideram ideal um genótipo com coeficiente de regressão igual a um com o menor desvio de regressão possível. Estudos de estabilidade e adaptabilidade do algodoeiro por meio de metodologias baseadas na análise de regressão foram realizados em várias ocasiões (Farias et al., 1997; Suinaga et al., 2006; Hoogerheid et al., 2007).

Lin \& Binns (1988) propuseram uma medida que representa a superioridade de um genótipo para o conjunto de ambientes avaliados. A estatística $P_{i}$ sugerida pelos autores mede o desempenho de um dado genótipo em relação ao genótipo com melhor desempenho, em cada um dos ambientes avaliados, e considera-se o genótipo mais promissor o que apresentar menor estimativa de $\mathrm{P}_{\mathrm{i}}$. Farias et al. (1997) verificaram similaridade entre essa metodologia e a proposta por Eberhart \& Russell (1966) em estudo de adaptabilidade do algodoeiro.

Princípio similar ao anterior foi proposto por Annicchiarico (1992), e estima o índice de confiança de determinado genótipo apresentar desempenho abaixo ou acima da média do ambiente. Essa análise foi aplicada por Machado et al. (2003) e Mora et al. (2007) em estudos de adaptabilidade e estabilidade do algodoeiro, sendo que os primeiros autores analisaram características tecnológicas de fibras e os últimos a produtividade.

Nos modelos mencionados até agora, estimativas de parâmetros de estabilidade similares para diferentes genótipos não implicam, necessariamente, um mesmo padrão de resposta às variações do ambiente: genótipos podem ser adaptados a ambientes específicos e, em média, apresentarem a mesma estimativa de estabilidade ou de índices de superioridade. O modelo AMMI ("additive main effects and multiplicative interaction") (Zobel et al., 1988) pode contornar essa limitação. Nesse modelo, por meio de uma análise gráfica, em biplot, busca-se identificar, simultaneamente, padrões de interação para genótipos e ambientes. Na cultura do algodoeiro, esse modelo foi utilizado por Souza et al. (2006).

Diante da diversidade de modelos para estudo da interação GxA e da importância desse fenômeno para a cotonicultura, este trabalho teve como objetivo comparar diferentes métodos para avaliar a estabilidade e a adaptabilidade do caráter produtividade de algodão em caroço em 17 genótipos de algodoeiro no Cerrado brasileiro.

\section{Material e Métodos}

A produtividade de algodão em caroço foi avaliada em 17 genótipos de algodoeiro em 23 ambientes do Cerrado brasileiro na safra 2005/2006. Todos os ensaios foram delineados em blocos completos ao acaso com quatro repetições. As parcelas foram constituídas por quatro linhas de $5 \mathrm{~m}$, com as duas fileiras centrais como área útil.

Os ambientes de avaliação estavam dispostos do seguinte modo: três na Bahia, em São Desidério (A1), Correntina (A2) e Barreiras (A3); sete em Goiás, em Ipameri (A4), Itumbiara (A5), Montividiu (A6), Palmeiras (A7), Santa Helena (A8), Paraúna (A9) e Perolândia (A10); seis no Mato Grosso, em Campo Verde (A11), Lucas do Rio Verde (A12), Novo São Joaquim (A13), Serra da Petrovina (A14), Primavera do Leste (A15) e Rondonópolis (A16); seis no Mato Grosso do Sul, em Aral Moreira 1 (A17), Aral Moreira 2 (A18), Chapadão do Céu (A19), Dourados (A20), Itaquari (A21) e Nioaque (A22); e um no Piauí, em Bom Jesus (A23).

As seguintes cultivares ou linhagens foram avaliadas: BRS Aroeira (G1), BRS Cedro (G2), BRS Araçá (G3), BRS 269 - Buriti (G4), CNPA GO 2001-999 (G5), Fibermax 966 (G6), Fibermax 977 (G7), CNPA CO 200156818 (G8), Delta Opal (G9), SL 506 (G10), FMT 701 (G11), Coodetec 406 (G12), Coodetec 409 (G13), Fabrika (G14), Delta Penta (G15), BRS Ipê (G16), CNPA CO 2000337 (G17).

O manejo cultural variou conforme os ambientes de avaliação. Nas situações em que os experimentos foram implantados em áreas de lavoura comercial, adotou-se o manejo utilizado na própria fazenda. O espaçamento entre linhas variou conforme o utilizado em cada região.

Foram realizadas análises de variâncias individuais e a análise conjunta, conforme descrito em Ramalho et al. (2000). A razão entre o maior e o menor quadrado médio 
residual, entre os obtidos em todos os ambientes, foi inferior a sete e, portanto, consideraram-se os erros como homogêneos, para fins da análise conjunta (Gomes, 1987). As análises de adaptabilidade e estabilidade foram realizadas empregando-se as seguintes metodologias:

a) Ecovalência - proposta por Wrick (1965), citado por Cruz \& Regazzi (1997), pela qual a ecovalência de cada cultivar é estimada por:

$\mathrm{W}_{\mathrm{i}}=\sum_{\mathrm{j}=1}^{\mathrm{n}}\left(\mathrm{Y}_{\mathrm{ij}}-\overline{\mathrm{Y}}_{\mathrm{i}}-\overline{\mathrm{Y}}_{\mathrm{j}}+\overline{\mathrm{Y}}_{\ldots .}\right)^{2}$, em que $\mathrm{Y}_{\mathrm{ij}}$ é a média do genótipo i no ambiente j; $\bar{Y}_{\mathrm{i}}$ é a média do genótipo i; $\bar{Y}_{. j}$ é a média do ambiente $\mathrm{j} ; \bar{Y}_{\text {.. }}$ é a média geral; $\mathrm{n}$ é o número de ambientes.

b) Eberhart \& Russell (1966) - o modelo de regressão linear para essa metodologia é:

$\mathrm{Y}_{\mathrm{ij}}=\mathrm{m}_{\mathrm{i}}+\mathrm{b}_{\mathrm{i}} \mathrm{I}_{\mathrm{j}}+\mathrm{d}_{\mathrm{ij}}+\overline{\mathrm{e}}_{\mathrm{ij}}$, em que $\mathrm{Y}_{\mathrm{ij}}$ é a média observada do genótipo i no ambiente $\mathrm{j} ; \mathrm{m}_{\mathrm{i}}$ é a média geral do genótipo i; $b_{i}$ é o coeficiente de regressão do genótipo i; $\mathrm{I}_{\mathrm{j}}$ é o índice ambiental $\mathrm{j}$; $\mathrm{d}_{\mathrm{ij}}$ é o desvio da regressão do genótipo i no ambiente $\mathrm{j} ; \overline{\mathrm{e}}_{\mathrm{ij}}$ é o erro médio associado à média. O índice ambiental é calculado por $\mathrm{I}_{\mathrm{j}}=\overline{\mathrm{Y}}_{\mathrm{j}}-\overline{\mathrm{Y}}_{\mathrm{.}}$, com $\sum_{\mathrm{j}=1}^{\mathrm{n}} \mathrm{I}_{\mathrm{j}}=0$, em que $\overline{\mathrm{Y}}_{. .}$é a média geral; $\overline{\mathrm{Y}}_{\mathrm{j}}$ é a média do ambiente j; n é o número de ambientes.

Como critério de previsibilidade de cada genótipo (estabilidade), adotou-se o coeficiente de determinação da equação de regressão $\left(\mathrm{R}^{2}\right)$ superior a $80 \%$ (Cruz \& Regazzi, 1997).

c) Lin \& Binns (1988) - adota-se a medida $\mathrm{P}_{\mathrm{i}}$ como a distância quadrática média entre o genótipo i e o genótipo com resposta máxima no ambiente $\mathrm{j}$, da seguinte forma:

$P_{i}=\sum_{j=i}^{n}\left(Y_{i j}-M_{j}\right)^{2} / 2 n$, em que $P_{i}$ é o índice de superioridade do genótipo i; $\mathrm{Y}_{\mathrm{ij}}$ é a produtividade do genótipo i no ambiente $\mathrm{j} ; \mathrm{M}_{\mathrm{j}}$ é a produtividade do genótipo com resposta máxima entre todos os genótipos no ambiente j; n é o numero de ambientes. Essa expressão pode ser desdobrada em:

$P_{i}=\left[n\left(Y_{i}-\bar{M}\right)^{2}+\sum_{j=1}^{n}\left(Y_{i j}-\bar{Y}_{i}-M_{j}+\bar{M}\right)^{2}\right] / 2 n$ em que $\overline{Y_{i} .}$ é a

média do genótipo i e $\overline{\mathrm{M}}$ é a média dos genótipos com resposta máxima. Considerando que $\mathrm{M}_{\mathrm{j}}$ representa um genótipo hipotético, então o primeiro termo da equação representa a soma de quadrados para o efeito genético e o segundo, a soma de quadrados da interação genótipos $\mathrm{x}$ ambientes .

d) Annicchiarico (1992) - nessa metodologia o índice de confiança $\mathrm{I}_{\mathrm{i}}$ para cada cultivar é dado por:
$\mathrm{I}_{\mathrm{i}}=\overline{\mathrm{Y}}_{\mathrm{i}}-\mathrm{Z}_{(1-\alpha)} \mathrm{S}_{\mathrm{i}}$ em que: $\mathrm{I}_{\mathrm{i}}$ é o índice de confiança (\%); $\bar{Y}_{i}$. é a média geral do genótipo i em porcentagem; $Z$ é o percentil $(1-\alpha)$ da função de distribuição normal acumulada; $\alpha$ é o nível de significância; $\mathrm{S}_{\mathrm{i}}$ é o desviopadrão dos valores percentuais. Neste trabalho dois índices de confiança foram adotados, 75 e $95 \%$.

e) Modelo AMMI - compreende componentes aditivos, para os efeitos principais (genótipos e ambientes), e componentes multiplicativos, para a interação. Os efeitos dos desvios da interação são analisados pela técnica multivariada de componentes principais e decomposição de valores singulares. A variação contida nos componentes significativos é chamada de "padrão", enquanto a contida nos não-significativos, de "ruído". A interpretação da adaptabilidade e establilidade foi realizada por meio de análise gráfica, em biplot, dos dois primeiros componentes principais. Genótipos e ambientes mais próximos à origem do biplot são os mais estáveis; em contraponto, aqueles mais afastados da origem são os que mais contribuem para a interação. Genótipos ou ambientes próximos entre si apresentam o mesmo padrão de comportamento para a interação GxA. Genótipos e ambientes presentes em um mesmo quadrante potencializam o efeito da interação GxA. Maiores detalhes sobre o modelo são apresentados em Duarte \& Vencovsky (1999) e em Dias (2005).

\section{Resultados e Discussão}

Os resultados da análise de variância conjunta são apresentados na Tabela 1. Todas as fontes de variação foram significativas, indicando que os genótipos têm diferentes potenciais produtivos e que os ambientes de avaliação também diferem quanto às condições edafoclimáticas ou de manejo cultural que influenciam o desenvolvimento e produtividade do algodoeiro. A significância da interação GxA indica, ainda, que os genótipos têm comportamento não consistente nos ambientes.

Tabela 1. Resumo da análise de variância conjunta quanto à produção de algodão em caroço $\left(\mathrm{kg} \mathrm{ha}^{-1}\right)$, de 17 genótipos avaliados em 23 ambientes, no Cerrado brasileiro.

\begin{tabular}{lrrrr}
\hline Fonte de variação & \multicolumn{1}{c}{ GL } & QM & \multicolumn{1}{c}{ Fc } & Pr>Fc \\
\hline Genótipos (G) & 16 & $6.874 .445,8$ & 26,4 & 0,000 \\
Ambientes (A) & 22 & $8.510 .230,2$ & 327,8 & 0,000 \\
GxA & 352 & $866.775,0$ & 3,3 & 0,000 \\
\hline Resíduo & 1.104 & $260.888,5$ & & \\
\hline
\end{tabular}


A produção média de algodão em caroço variou de $2.048 \mathrm{~kg} \mathrm{ha}^{-1}$, em Primavera do Leste (A15), a $5.449 \mathrm{~kg} \mathrm{ha}^{-1}$, em Campo Verde (A11), sendo a média geral de $3.747 \mathrm{~kg} \mathrm{ha}^{-1}$. Os genótipos que apresentaram maior e menor estimativa de produtividade, na média dos locais, foram BRS 269 - Buriti e BRS Ipê, com 4.185 e $3.149 \mathrm{~kg} \mathrm{ha}^{-1}$, respectivamente (Tabela 2).

Considerando-se o conceito de estabilidade preconizado pela metodologia da ecovalência, admitese como genótipo mais estável a cultivar BRS Cedro que contribuiu com apenas $2,12 \%$ para a interação. Com menor estabilidade, podem ser citadas as cultivares Delta Penta e BRS Ipê, que contribuíram com 17,6 e $15,7 \%$ para a interação $\mathrm{GxA}$, respectivamente (Tabela 3). Contudo, as três cultivares produziram abaixo da média geral. As duas cultivares mais produtivas, BRS 269 - Buriti e FMT 701, contribuíram com 5,03 e $4,25 \%$ para a interação, respectivamente.

A correlação de Spearman entre a produtividade média e a ecovalência foi de -0,086 (Tabela 4), sem evidência, portanto, de que a estabilidade fenotípica, mensurada pela contribuição dos genótipos para interação, esteja associada ao potencial produtivo médio das cultivares.

A maioria dos genótipos apresentou alta previsibilidade de comportamento pela metodologia de Eberhart \& de Cruz \& Regazzi (1997). As exceções foram as cultivares Delta Penta e BRS Ipê, com estimativas de $\mathrm{R}^{2}$ de 58,2 e $61,4 \%$, respectivamente (Tabela 3 ). Esses resultados estão coerentes com os obtidos por meio da ecovalência, em que esses dois genótipos foram os que mais contribuíram para a interação GxA. No outro extremo, essa verificação também é verdadeira; os quatro genótipos com maiores estimativas de $\mathrm{R}^{2}$ também apresentaram as menores estimativas de ecovalência. A correlação de Spearman entre as estimativas de $\mathrm{R}^{2} \mathrm{e}$ de ecovalência foi - 0,936 (Tabela 4), o que reafirma que quanto maior a contribuição da cultivar para a interação, menor a sua previsibilidade.

Os parâmetros obtidos por essa metodologia para a cultivar BRS Ipê foram contrastantes com os obtidos por Suinaga et al. (2006), no estado do Mato Grosso. Esses autores observaram que essa cultivar apresentouse como produtiva, adaptada e estável, embora em uma rede de ensaios mais restrita. Hoogerheid et al. (2007) encontraram estimativas de $\mathrm{R}^{2}$, para a cultivar BRS Aroeira, igual a 96, e de 74\%, para a cultivar Delta Opal. Tais diferenças podem ser explicadas, em parte, Russell (1966), quando avaliados pelo critério $\mathrm{R}^{2}>80 \%$,

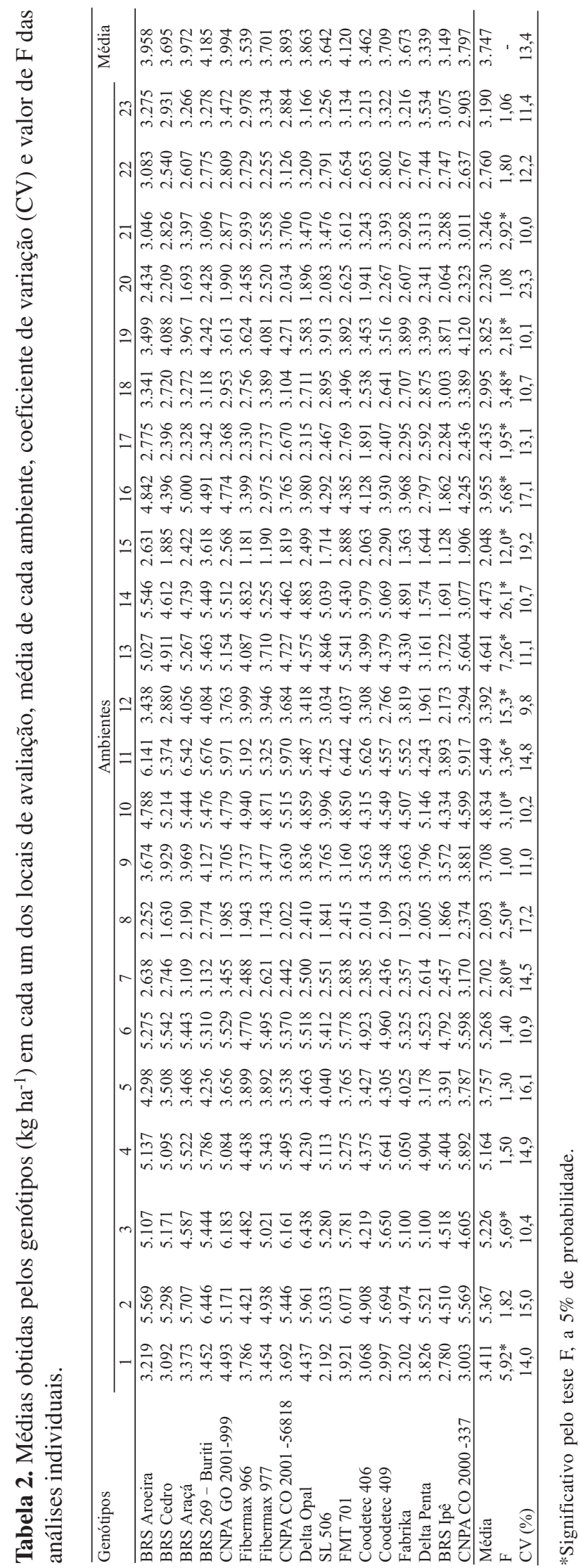

Pesq. agropec. bras., Brasília, v.43, n.3, p.349-355, mar. 2008 
pelo fato de o índice ambiental ser dependente dos genótipos que estão sendo avaliados e, como esses variam entre os experimentos, alterações nos resultados obtidos devem ser esperadas.

Observa-se que três dos 17 genótipos (BRS Cedro, BRS Araçá, CNPA CO 2001-56818) apresentaram coeficiente de regressão $b_{i}$ estatisticamente superior a um, o que indica adaptabilidade a ambientes favoráveis (Tabela 3). Outrossim, BRS Araçá e CNPA CO 200156818 produziram acima da média geral e, portanto, foram mais apropriadas para ambientes melhorados do que a BRS Cedro. De fato, o desempenho máximo da BRS Araçá (6.542 $\mathrm{kg} \mathrm{ha}^{-1}$ ) foi alcançado em Campo Verde (A11), ambiente de maior média, no qual a cultivar revelou-se mais produtiva. Já para a linhagem CNPA CO 2001-56818, o máximo desempenho foi obtido em Barreiras, BA (A3), sob irrigação, com produtividade acima de $6.000 \mathrm{~kg} \mathrm{ha}^{-1}$ (Tabela 2).

Outras três cultivares (Fibermax 966, Delta Penta e BRS Ipê) apresentaram o coeficiente $b_{i}$ inferior a um, indicando adaptação a ambientes desfavoráveis. Porém, suas médias foram inferiores à média geral e, portanto, possuem capacidade produtiva abaixo da média. Mesmo em ambientes desfavoráveis, como Primavera do Leste (A15) e Santa Helena (A8), elas produziram abaixo da média desses ambientes. Contudo, os dados de avaliação correspondem apenas a uma safra e devem ser vistos com ressalvas.
Quanto aos demais genótipos, as estimativas de $\mathrm{b}_{\mathrm{i}}$ foram estatisticamente iguais a um e, portanto, com adaptabilidade geral aos ambientes. As cultivares BRS 269 - Buriti, FMT 701 e o genótipo CNPA GO 2001-999 são os mais aproximados do ideal estabelecido por Eberhart \& Russell (1966). Tais genótipos foram os três mais produtivos, apresentaram coeficiente $b_{i}$ estatisticamente igual a um e $\mathrm{R}^{2}$ superiores a $80 \%$, o que thes confere alta estabilidade ou previsibilidade, e seriam os materiais mais recomendados para plantio no Cerrado, de acordo com essa metodologia.

Resultados similares foram obtidos quando se tomou por referência a medida de superioridade geral estabelecida por Lin \& Binns (1988). Nesse caso, os genótipos mais adaptados foram BRS 269 - Buriti e FMT 701 (Tabela 3). Contudo, considerando o ponto de corte, apenas a cultivar BRS 269 - Buriti não diferiu estatisticamente do máximo $(\mathrm{p}<0.05)$. Os genótipos menos adaptados foram Delta Penta e BRS Ipê. Ao se analisar a contribuição de cada genótipo para a interação GxA, de acordo com a metodologia de Lin \& Binns (1988), observa-se que a 'BRS 269 - Buriti' contribuiu com menos de $2 \%$, enquanto as menos adaptadas contribuíram com 16,9\% (Delta Penta) e 18,06\% (BRS Ipê), corroborando os resultados obtidos pelas metodologias da ecovalência e de Eberhart \& Russell (1966). Em virtude da similaridade entre as metodologias, considera-se o uso do $\mathrm{P}_{\mathrm{i}}$ vantajoso pela facilidade de aplicação e interpretação.

Tabela 3. Estimativas dos parâmetros de estabilidade das metodologias de Eberhart \& Russell (1966), Ecovalência (Wrick, 1965) e Lin \& Binns (1988), para 17 genótipos de algodão, avaliados em 23 ambientes do Cerrado brasileiro.

\begin{tabular}{|c|c|c|c|c|c|c|c|c|}
\hline \multirow[t]{2}{*}{ Cultivares } & \multicolumn{3}{|c|}{ Eberhart e Russell } & \multirow{2}{*}{$\begin{array}{l}\text { Wrick } \\
\mathrm{W}_{\mathrm{i}}^{2}(\%)\end{array}$} & \multicolumn{2}{|c|}{ Lin e Binns } & \multicolumn{2}{|c|}{ Annicchiarico } \\
\hline & Médias & $\mathrm{b}_{\mathrm{i}}$ & $\mathrm{R}^{2}$ & & $\mathrm{P}(\mathrm{i})$ & $\%($ gxe $)$ & $\mathrm{I}_{75}$ & $\mathrm{I}_{95}$ \\
\hline BRS Buriti (G4) & 4.185 & $1,033^{\mathrm{ns}}$ & 88,6 & 5,03 & $386.711,6$ & 1,97 & 101,7 & 85,6 \\
\hline FMT 701 (G11) & 4.120 & $1,082^{\mathrm{ns}}$ & 91,4 & 4,25 & $436.326,3$ & 1,60 & 102,9 & 92,0 \\
\hline CNPA GO 2001-999 (G5) & 3.994 & $1,074^{\mathrm{ns}}$ & 88,3 & 5,77 & $704.521,2$ & 2,10 & 97,9 & 85,5 \\
\hline BRS Araçá (G3) & 3.972 & $1,116^{*}$ & 90,5 & 5,23 & $922.112,6$ & 3,79 & 97,7 & 86,6 \\
\hline BRS Aroeira $(\mathrm{G} 1)$ & 3.958 & $1,004^{\mathrm{ns}}$ & 90,8 & 3,71 & $847.620,5$ & 2,77 & 99,5 & 89,8 \\
\hline CNPACO 2001-56818 (G8) & 3.893 & $1,141^{*}$ & 95,2 & 3,11 & $1.096 .095,9$ & 3,76 & 97,0 & 88,5 \\
\hline Delta Opal (G9) & 3.863 & $1,037^{\mathrm{ns}}$ & 86,6 & 6,08 & $1.105 .204,2$ & 3,13 & 94,9 & 83,0 \\
\hline CNPA CO 2000-337 (G17) & 3.797 & $1,030^{\mathrm{ns}}$ & 85,1 & 6,77 & $1.725 .742,3$ & 7,50 & 93,5 & 82,1 \\
\hline Coodetec $409(\mathrm{G} 13)$ & 3.709 & $0,994^{\mathrm{ns}}$ & 89,6 & 4,16 & $1.650 .996,1$ & 4,26 & 92,8 & 83,6 \\
\hline Fibermax 977 (G7) & 3.701 & $1,001^{\mathrm{ns}}$ & 85,4 & 6,20 & $2.148 .019,2$ & 8,85 & 88,4 & 74,2 \\
\hline BRS Cedro (G2) & 3.695 & $1,109 *$ & 96,3 & 2,12 & $1.584 .945,8$ & 3,18 & 91,9 & 83,9 \\
\hline Fabrika (G14) & 3.673 & $1,024^{\mathrm{ns}}$ & 94,5 & 2,25 & $1.819 .151,8$ & 4,75 & 90,7 & 81,0 \\
\hline SL $506($ G10) & 3.642 & $0,999^{\mathrm{ns}}$ & 88,5 & 4,69 & $2.096 .567,3$ & 6,44 & 89,5 & 79,1 \\
\hline Fibermax 966 (G6) & 3.539 & $0,883^{\mathrm{ns}}$ & 86.6 & 4,87 & $2.547 .086,9$ & 7,26 & 86,4 & 74,5 \\
\hline Coodetec 406 (G12) & 3.462 & $0,908^{\mathrm{ns}}$ & 93.4 & 2,42 & $2.486 .845,6$ & 3,70 & 87,8 & 81,0 \\
\hline Delta Penta (G15) & 3.339 & $0,781 * *$ & 58.2 & 17,60 & $4.380 .132,8$ & 16,90 & 77,8 & 59,7 \\
\hline BRS Ipê (G16) & 3.149 & $0,785 * *$ & 61.4 & 15,70 & $5.444 .780,3$ & 18,06 & 72,4 & 55,0 \\
\hline Ponto de corte $\mathrm{P}(\mathrm{i})$ & 404.832 & & & & & & & \\
\hline
\end{tabular}

$*$ e **Significativo a 5 e $1 \%$ de probabilidade, respectivamente, pelo teste t. 
É preciso ressaltar que o índice $P_{i}$ mede desvios em relação aos genótipos de melhor desempenho em cada ambiente, e não uma reposta à melhoria das condições ambientais, como na metodologia de Eberhart \& Russell (1966). A correlação de Spearman entre as médias dos genótipos e os índices $\mathrm{P}_{\mathrm{i}}$ foi de $-0,966$ (Tabela 4), indicando que o ordenamento dos genótipos oscilou pouco ao longo dos ambientes. Ou seja, no conjunto de dados analisados, a média dos materiais em estudo seria um excelente critério de seleção.

Os resultados obtidos pela metodologia de Annicchiarico (1992) foram muito similares aos obtidos pelo modelo de Lin \& Binns (1988). A correlação de Spearman entre os índices $\mathrm{P}_{\mathrm{i}}$ e $\mathrm{I}_{75}$ foi -0,985 (Tabela 4). Esses resultados estão de acordo com os obtidos por Mora et al. (2007) e Machado et al. (2003), que também encontraram resultados concordantes pelos dois modelos; os primeiros autores estudaram a estabilidade e adapatabilidade para produtividade de algodão, enquanto os últimos, as características tecnológicas de fibras. A similaridade entre as metodologias de Lin \& Binns (1988) e Annicchiarico (1992) é esperada. Ambas têm por finalidade medir a superioridade dos genótipos: a primeira toma como referência o desempenho dos melhores genótipos em cada ambiente e a segunda a média de cada um dos ambientes.

As cultivares FMT 701 e BRS 269 - Buriti são as que têm $75 \%$ de chance $\left(I_{75}\right)$ de, em última instância, produzir acima da média ambiental (Tabela 3 ). O fato de explicitar o potencial produtivo e estabelecer o risco da escolha de determinada cultivar facilita a interpretação da metodologia proposta por Annicchiarico (1992).

Em adição, conforme o nível de confiança estabelecido, a classificação dos genótipos pode ser alterada, visto que o desvio-padrão dos índices é específico para cada genótipo. Quando se considera um índice de confiança igual a $95 \%$, observa-se que a cultivar BRS 269 - Buriti, a mais produtiva na média

Tabela 4. Correlações classificatórias de Spearman entre os parâmetros de estabilidade e adaptabilidade das diferentes metodologias.

\begin{tabular}{lcccccc}
\hline Variável & $\mathrm{B} 1(\mathrm{i})$ & $\mathrm{R}^{2}$ & $\mathrm{Wi}(\%)$ & $\mathrm{P}(\mathrm{i})$ & $\mathrm{I}_{75}$ & $\mathrm{I}_{95}$ \\
\hline Média & 0,745 & 0,297 & $-0,086$ & $-0,966$ & 0,973 & 0,875 \\
B1(i) $^{2}$ R $^{2}$ & & 0,561 & $-0,312$ & $-0,775$ & 0,748 & 0,782 \\
Wi (\%) & & & $-0,936$ & $-0,457$ & 0,411 & 0,616 \\
P(i) & & & & 0,260 & $-0,216$ & $-0,426$ \\
I $_{75}$ & & & & & $-0,985$ & $-0,931$ \\
\hline
\end{tabular}

geral, seria a quinta colocada entre as mais adaptadas; enquanto a BRS Aroeira, quinta no ordenamento pelas produtividades, seria a segunda, no ordenamento das mais adaptadas (Tabela 3).

Com relação ao desdobramento dos efeitos da interação GxA original, constatou-se que os dois primeiros termos multiplicativos do modelo AMMI foram significativos. Juntos, estes termos (primeiro e segundo componentes principais) explicaram 55\% da variação total da interação.

Os resultados verificados pelo AMMI corroboram os obtidos pela ecovalência, e ratificam a constatação de que as cultivares Delta Penta (G15) e BRS Ipê (G16) foram as que mais contribuíram para a interação (Figura 1). Essas cultivares possuíram o mesmo padrão de interação, já que encontram-se muito próximas no biplot. Essa informação é uma das vantagens do uso do AMMI em relação à ecovalência, visto que essa última apenas mensura a contribuição de cada genótipo para a interação, sem especificar o padrão de comportamento em relação aos demais. Entre as mais estáveis, por essa metodologia, estão as cultivares BRS Cedro (G2) e SL 506 (G10), ambas muito próximas à origem do biplot.

A representação gráfica permite tanto a identificação de genótipos estáveis, com ampla adaptabilidade, quanto de adaptabilidades específicas, o que pode ser interessante em estudos que abordam ampla gama de ambientes, tal como na vasta condição de Cerrado. Na Figura 1, é possível verificar adaptabilidades específicas entre CNPA GO 2001-999 (G5) e Lucas do Rio Verde (A12); entre BRS 269 - Buriti (G4) e Campo Verde (A11); entre BRS Araçá (G3) e Novo São Joaquim (A13).

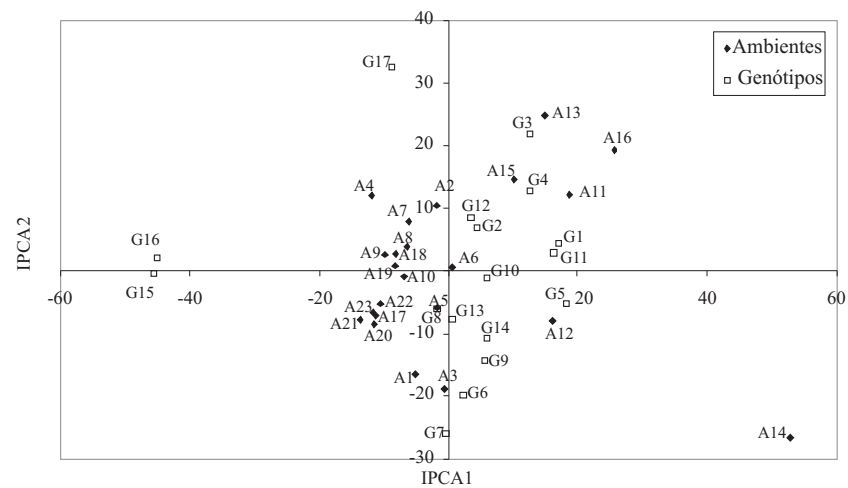

Figura 1. Biplot AMMI2 para 17 genótipos de algodoeiro herbáceo (G1 a G17) e 23 ambientes (A1 a A23). 


\section{Conclusões}

1. As metodologias de Eberhart \& Russell, Lin \& Binns e Annicchiarico são concordantes, e identificam genótipos de alta adaptabilidade e elevado desempenho produtivo.

2. A metodologia AMMI permite a verificação de adaptabilidade específica, de grande valia para fins de zoneamento e indicações específicas, em estudos com vasto conjunto de ambientes.

3. Pela facilidade de uso e interpretação, é conveniente o uso combinado das metodologias de Lin \& Binns e AMMI.

\section{Referências}

ANNICCHIARICO, P. Cultivar adaptation and recommendation from alfafa trials in Northern Italy. Journal of Genetics and Breeding, v.46, p.269-278, 1992.

CRUZ, C.D.; REGAZZI, A.J. Modelos biométricos aplicados ao melhoramento genético. 2.ed. Viçosa: UFV, 1997. 390p.

DIAS, C.T.S. Métodos para escolha de componentes de efeito principal aditivo e interação multiplicativa (AMMI). 2005.73p. Tese (Livre Docência) - Escola Superior de Agricultura Luiz de Queiroz, Piracicaba.

DUARTE, J.B.; VENCOVSKY, R. Interação genótipos x ambientes: uma introdução à análise "AMMI". Ribeirão Preto: Sociedade Brasileira de Genética, 1999. 60p. (Séries Monografias, 9).

EBERHART, S.A.; RUSSELL, W.A. Stability parameters for comparing varieties. Crop Science, v.6, p.36-40, 1966.

FARIAS, F.J.C. Índice de seleção em cultivares de algodoeiro herbáceo. 2005. 121p. Tese (Doutorado) - Escola Superior de Agricultura Luiz de Queiroz, Piracicaba.
FARIAS, F.J.C.; RAMALHO, M.A.P.; CARVALHO, L.P.; MOREIRA, J.A.N.; COSTA, J.N. Parâmetros de estabilidade propostos por Lin e Binns (1988) comparados com o método da regressão. Pesquisa Agropecuária Brasileira, v.32, p.407-414, 1997.

GOMES, F.P. Curso de estatística experimental. 11.ed. Piracicaba: Nobel, 1987. 466p.

HOOGERHEID, E.S.S.; FARIAS, F.J.C.; VENCOVSKY, R.; FREIRE, E.C. Estabilidade fenotípica de genótipos de algodoeiro no Estado do Mato Grosso. Pesquisa Agropecuária Brasileira, v.42, p.695-698, 2007.

LIN, C.S.; BINNS, M.R. A superiority measure of cultivar performance for cultivar $x$ location data. Canadian Journal of Plant Science, v.68, p.193-198, 1988.

MACHADO, J.R.A.; PENNA, J.C.V.; FALLIERI, J.; SANTOS, P.G.; LANZA, M.A. Adaptabilidade e estabilidade de genótipos de algodoeiro para características tecnológicas de fibra. Revista Brasileira de Oleaginosas Fibrosas, v.7, p.673-683, 2003.

MORA, F.; PUPIM-JUNIOR, O.; SCAPIM, C.A. Prediction of cultivar effects on cotton yield in the presence of genotypeenvironment interaction. Ciencia e Investigación Agraria, Santiago, v.34, p.7-16, 2007.

RAMALHO, M.A.P.; FERREIRA, D.; OLIVEIRA, A.C. Experimentação em genética e melhoramento de plantas. Lavras: Ufla, 2000. 326p.

RAMALHO, M.A.P.; SANTOS, J.B. dos; ZIMMERMANN, M.J. de O. Genética quantitativa em plantas autógamas: aplicações ao melhoramento do feijoeiro. Goiânia: UFG, 1993. 271p.

SOUZA, A.A.; FREIRE, E.C.; BRUNO, R.L.A.; CARVALHO, L.P.; SILVA FILHO, J.L.; PEREIRA, W.E. Estabilidade e adaptabilidade do algodoeiro herbáceo no Cerrado do Mato Grosso e Mato Grosso do Sul. Pesquisa Agropecuária Brasileira, v.41, p.1125-1131, 2006.

SUINAGA, F.A.; BASTOS, C.S.; RANGEL, L.E.P. Fenotipic adaptability and stability of cotton cultivars in Mato Grosso State, Brazil. Pesquisa Agropecuária Tropical, v.36, p.145-150, 2006.

ZOBEL, R.W.; WRIGHT, M.J.; GAUCH JÚNIOR, H.G. Statistical analysis of a yield trial. Agronomy Journal, v.80, p.388-393, 1988.

Recebido em 19 de novembro de 2007 e aprovado em 18 de fevereiro de 2008 